Check for updates

Cite this: Phys. Chem. Chem. Phys., 2019, 21, 13526

Received 28th March 2019 Accepted 7th May 2019

DOI: $10.1039 / c 9 c p 01746 a$

rsc.li/pccp

\section{On the magnetosensitivity of lipid peroxidation: two- versus three-radical dynamics $\dagger$}

\author{
Chris Sampson, Robert H. Keens and Daniel R. Kattnig (iD *
}

\section{Introduction}

Most chemical reactions are insensitive to the weak magnetic fields that pervade our everyday surroundings. ${ }^{1}$ An exception to this rule is the recombination of radical pairs, which depends on the overall electron spin of the reactants. ${ }^{1,2}$ As a consequence of weak magnetic interactions modulating the overall spin of the radicals, the associated recombination yields are, at least in principle, susceptible to the strength and direction of external magnetic fields. ${ }^{1,3-6}$ The so-called radical pair mechanism (RPM) provides a comprehensive framework for the theoretical description of the spin dynamics that underpin these processes. ${ }^{1,2,4,7-12}$ The resulting magnetic field effects (MFEs) have been widely studied, both experimentally and theoretically. ${ }^{13-16}$ The RPM is the leading explanation for certain forms of animal magnetoreception, with the avian migratory compass being a notable example..$^{4,17-19}$ Unexpectedly, in view of its reigning in a hot and noisy biological environment, truly quantum effects have been suggested to reinforce the sensitivity of this compass. ${ }^{19-21}$ This observation makes the RPM an important contender in the emerging field of quantum biology. ${ }^{22}$

Living Systems Institute and Department of Physics, University of Exeter, Stocker Road, Exeter, Devon, EX4 4QD, UK. E-mail: D.R.Kattnig@exeter.ac.uk

$\dagger$ Electronic supplementary information (ESI) available. See DOI: 10.1039/c9cp01746a
The RPM involves the formation of a radical pair, which can be geminate, i.e., formed in an elementary reaction, typically from diamagnetic precursors, or as an F-pair, i.e., resulting from a random encounter of radicals. ${ }^{1,2,4,7-12}$ Once formed, these radical pairs tend to be short-lived, but show reactivity that is controlled by spin dynamics. They can coherently interconvert between electronic singlet and triplet states under the action of magnetic interactions and inter-radical interactions such as the exchange coupling. Specifically, the mixing of the electron spin states can occur by the difference in the Zeeman precession frequency ( $\Delta g$ mechanism) or the interaction with magnetic nuclei within the radicals (hyperfine mechanism). It is most efficient if the singlet and triplet energy levels become quasi degenerate, e.g., for distances at which the exchange coupling has decayed. As an external magnetic field can impact the relative energy of the energy levels or, in the case of the $\Delta g$ mechanism, impact directly upon the mixing term, the population of the spin states and their coherent interconversion becomes magnetosensitive. As a result of spin-selective recombination reactions, these MFE on spin dynamics can eventually be reflected in product/recombination yields. More details are found in ref. 1 , 2, 4 and 7-12.

Remarkable magnetosensitive phenomena can also emerge from systems comprising three radicals instead of the conventional radical pair. If the dominant interaction among the radicals is their 
exchange coupling, this leads to the field of spin catalysis, which has been reviewed in ref. 23. If, on the other hand, the function of the third radical is predominantly to scavenge one of the radicals of the original pair in a spin-selective side reaction, the "chemical Zeno effect" becomes observable, which has recently been hypothesized to boost the sensitivity of the avian magnetic compass. ${ }^{24-26}$ As the scavenging reaction immunizes the dynamics to fast spin relaxation in one of the radicals, this scavenging mechanism provides the realistic prospect of MFEs from systems involving swiftly relaxing species, such as superoxide, which, in radical pair reactions, preclude sensitivity to magnetic fields of moderate intensities (i.e., the mT-range). ${ }^{25,27,28}$ Recently, we have shown that, in systems of three or more radicals, MFEs can also result from the mutual electron-electron dipolar coupling alone. ${ }^{29}$ This is noteworthy as this process could, thus, elicit MFEs in the absence of hyperfine interactions and differences in radical $g$-factors, which are the corner stones of the dyadic RPM. Furthermore, the MFE persists in this new formalism for fields comparable to that of the geomagnetic field. For convenience of the subsequent discussion, we will henceforth refer to the dipolar coupling mechanism in three-radical systems as D3M.

We have previously suggested that the D3M could underpin the putative magnetosensitivity of lipid autoxidation, i.e., the oxidative degradation of lipids. ${ }^{29}$ Lipid peroxidation proceeds by a free-radical chain mechanism that involves peroxyl radicals as the main chain carriers. ${ }^{30-35}$ The chain reaction is initiated by abundant oxidising agents such as reactive oxygen species (ROS), which include superoxide $\mathrm{O}_{2}{ }^{\bullet-}$ and the hydroxyl radical $\mathrm{OH}^{\bullet} .^{36-39}$ The latter species can be formed through the Fenton reaction from hydrogen peroxide $\mathrm{H}_{2} \mathrm{O}_{2}{ }^{40}$ The reaction scheme shown in Fig. 1A, summarizes the most important reactions that account for initiation, propagation, termination and degenerate chain branching. ${ }^{32}$ Due to the ease of abstracting bis-allylic hydrogen atoms, poly un-saturated fatty acids are particularly susceptible to radical attack and, thus, are key to the process.

Many review articles describe lipid autoxidation in great detail and the reader is referred to those for a more in-depth discussion. ${ }^{30,41-43}$ Many experimental studies exist that have demonstrated that lipid autoxidation is sensitive to magnetic fields, and a correlation between MFE and levels of oxidative stress has been implicated. ${ }^{44-46}$ The body of evidence includes the level of lipid peroxidation in rats blood, ${ }^{34}$ brains, ${ }^{14}$ and lymphocytes, ${ }^{47,48}$ as well as guinea pigs, ${ }^{49}$ mouse brains, ${ }^{50,51}$ and in radish seeds. ${ }^{35} \mathrm{~A}$ few in vitro studies have been conducted on simpler model systems, yet the mechanistic details behind the results have remained elusive. Landoulsi et $a .^{52}$ have used bacterial strain $P$. aeruginosa and mutants thereof, and found that the level of lipid peroxidation increased when the bacteria were exposed to a $200 \mathrm{mT}$ static magnetic field. Takashima et al. ${ }^{53}$ found that exposing HL-60 cells to a combination of $\mathrm{H}_{2} \mathrm{O}_{2}$ and a weak ( $5 \mathrm{mT}$ ) magnetic field promotes cell death. Furthermore, it has been shown that by applying a steady magnetic field of $8 \mathrm{mT}$ to liposomes from 1,2-dioleoylphosphatidylcholine, the rate of lipid peroxidation can be increased. ${ }^{33}$ Kabuto et al. ${ }^{51}$ showed that, for field strengths between 2 and $4 \mathrm{mT}$, static magnetic fields actually had an inhibitory effect on iron-induced lipid peroxidation,
A
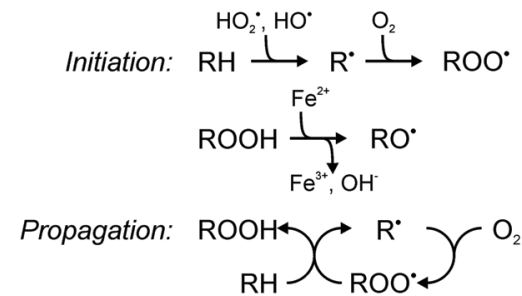

Chain branching: $2 \mathrm{ROOH} \longrightarrow \mathrm{ROO}^{*}+\mathrm{RO}^{*}+\mathrm{H}_{2} \mathrm{O}$

Termination: $2 \mathrm{ROO}^{\circ} \longrightarrow \mathrm{RO}_{4} \mathrm{R} \longrightarrow{ }^{1} \mathrm{O}_{2},{ }^{3} \mathrm{R}=\mathrm{O}, \mathrm{P}$

$2 \mathrm{R}^{\cdot} \longrightarrow \mathrm{P}^{\prime}$

$\mathrm{ROO}^{\circ}+\mathrm{R}^{*} \longrightarrow \mathrm{P}^{\prime \prime}$

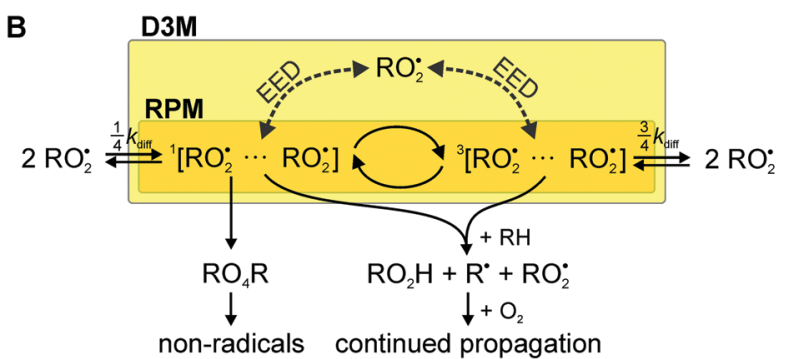

Fig. 1 (A) Key reaction steps in lipid peroxidation: initiation (I and II), propagation (III), degenerate-chain branching (IV) and termination (V-VII). The different Ps stand for diamagnetic termination products. $\mathrm{R}$ represents a lipid backbone. (B) The radical pair mechanism (orange section) specifically linked to lipid peroxidation in this example. The dashed arrows indicate the action of the EED interaction with a third radical, which may induce MFE via the D3M. $k_{\text {diff }}$ indicates the diffusive encounter rate constant. For random encounters of radicals, the ratio of triplet to singlet encounter is $3: 1$.

however, the autoxidation of linoleic acid was increased in a high field of $9.4 \mathrm{~T}^{54}$ These fields are evidently stronger than most of the weak background fields of our everyday surroundings. However, even background fields can cause an increase in oxidative stress. $^{55}$

As detailed in the reaction scheme in Fig. 1A, the chaincarrying peroxyl radicals are typically formed through the reaction of highly reactive carbon-centred radicals with molecular oxygen or through degenerate chain-branching (reactions III and IV in Fig. 1A). Although slow, the degenerate chain branching step gives way to interesting non-linear dynamics. If its rate approaches the rate of termination, a process, vividly described as chain ignition, can result in a strong increase in radical concentrations. ${ }^{56}$ Kipriyanov et al. ${ }^{57}$ have suggested that small alterations of the radical recombination efficiency could induce bifurcation transitions of bistable steady states. As a consequence, a small effect putatively exerted by magnetic fields on the chain branching reaction could, at least in principle, induce huge (by three orders of magnitude) changes in radical concentrations. It has been argued that the RPM cannot predict a MFE on the symmetric recombination of peroxyl radicals, because $\Delta g=0$ and no hyperfine interactions could be expected, as the spin density of peroxyl radicals centres on the terminal oxygen, for which all magnetic isotopes have insignificant natural abundance (for ${ }^{17} \mathrm{O}$, $\mathrm{I}=5 / 2$, but its abundance is only $0.037 \%$ ). Consequently, previous studies have associated magnetosensitivity with reactions other 
than the recombination of the most abundant peroxyl radicals. ${ }^{33,57,58}$ The same ideas appear to apply to the oxidation of hydrocarbons by molecular oxygen. ${ }^{58}$ Motivated by the evidence highlighted above, here we present a theoretical study of the RPM and the $\mathrm{D} 3 \mathrm{M}$ in the context of lipid autoxidation. For reasons that will become apparent below, we focus on the recombination of the main chain carriers, the lipid peroxyl radicals.

\section{The model}

The aim of this work is the assessment of the RPM and the D3M with respect to plausibility and relative importance. In particular, we provide a toy model, which aims to provide a first understanding, while (necessarily) neglecting many of the intricacies of true reactive radical encounters in membranes. We focus on the recombination of lipid peroxyl radicals (reaction (V)), because estimates based on established rate constants identify $\mathrm{RO}_{2}{ }^{\bullet}$ as the most abundant and long-lived (half-life of around $17 \mathrm{~s}$ ) radical species. ${ }^{59}$ Fig. 1B gives a schematic of the considered recombination process. The radicals are assumed to encounter as F-pairs, i.e., with a random initial spin configuration. Recombination in the singlet state can give rise to the formation of diamagnetic products via an unstable tetroxide intermediate (Russell mechanism, reaction (V) in Fig. 1A). ${ }^{60}$ The radicals can undergo singlet-triplet interconversion due to non-zero hyperfine interactions with magnetic nuclei (RPM) or the catalytic effect of a third radical interacting with the primary radical pair through the electron-electron dipolar interaction (D3M). If the inter-radical distance is small, this process will be influenced by the exchange interaction. ${ }^{29,61}$ These coherent processes potentially imprint a magnetic field sensitivity on the recombination yield.

Due to the spin density of the peroxyl radicals centering on the terminal oxygen, for which all magnetic isotopes have insignificant natural abundance (while for ${ }^{17} \mathrm{O} \mathrm{I}=5 / 2$ its abundance is only $0.037 \%$ ), the RPM has been deemed insufficient for the process shown in Fig. 1B. As a consequence, previous studies in this area have associated magnetosensitivity with reactions that do not involve the abundant peroxyl radicals. ${ }^{57,62}$

In more detail, our model is based on the following observations. We consider peroxyl radicals as rigid cylindrical bodies. Based on the known membrane area per lipid of $0.7 \mathrm{~nm}^{2}$ for 1-palmitoyl-2oleoyl-sn-glycero-3-phospholipids ${ }^{63}$ and a presumed hexagonal densest packing of the cylindrical lipids in the membrane (packing density of circles on a hexagonal lattice: $\pi \sqrt{3} / 6$ ), we estimate an effective lipid radius of $R=4.5 \AA$. Assuming furthermore that the hydrophobic peroxyl groups aggregate at the membrane interior, we can neglect the difference in vertical position of the radical functionalities across the membrane. This yields an essentially two-dimensional model of circular "peroxyl radicals" on the membrane plane.

We employ a simple Hamiltonian to calculate the singlet yield based on multiple parameters, such as the hyperfine, dipolar, exchange and Zeeman interactions. The Hamiltonian takes the form:

$$
\hat{H}=\hat{H}_{\mathrm{hfi}}+\hat{H}_{\mathrm{ze}}+\hat{H}_{\mathrm{dp}}+\hat{H}_{\mathrm{ex}}
$$

where $\hat{H}_{\mathrm{hfi}}$ represents the hyperfine interaction, $\hat{H}_{\mathrm{ze}}$ represents the Zeeman terms, $\hat{H}_{\mathrm{dp}}$ represents the electron-electron dipolar interaction between radicals and finally $\hat{H}_{\mathrm{ex}}$ is the Hamiltonian representing the exchange interaction. The interaction of the magnetic field with the nuclei is not included, as it is negligible compared to any of the considered interactions. The functional form for the hyperfine interaction is:

$$
\hat{H}_{\mathrm{hfi}}=\sum_{i=1}^{N} \sum_{j}^{n_{i}} a_{i j} \hat{\mathbf{I}}_{i j} \cdot \hat{\mathbf{S}}_{i},
$$

where $i$ labels a radical, $\hat{\mathbf{S}}_{i}$ is the spin vector operator for electron $i, N$ is the total number of radicals in the system, $n_{i}$ is the number of coupled nuclei in radical $i$ and $\hat{\mathbf{I}}_{i j}$ is the nuclear spin vector operator of nucleus $j$ in radical $i$. $a_{i j}$ is the related hyperfine coupling constant. The Zeeman Hamiltonian describes the interaction of the electron spins with the magnetic field and has the following form (in angular frequency units):

$$
\hat{H}_{\mathrm{ze}}=\omega \cdot \sum_{i=1}^{N} \hat{\mathbf{S}}_{i},
$$

where $\boldsymbol{\omega}$ is equal to $\frac{g \mu_{\mathrm{B}}}{\hbar} \mathbf{B}_{0}$ with $\mathbf{B}_{0}$ denoting the applied magnetic field. Here, we have assumed identical radicals, each of which is associated with the same $g$-factor of $g \approx 2 . \mu_{\mathrm{B}}$ is the Bohr magneton and $\hbar$ is the reduced Planck's constant.

The dipolar interaction between electrons is calculated by the point-dipolar approximation:

$$
\begin{gathered}
\hat{H}_{\mathrm{dp}}=\sum_{i<j}^{N} \hat{\mathbf{S}}_{i} \cdot \mathbf{D}_{i j} \cdot \hat{\mathbf{S}}_{j} \\
=d\left(\left|\mathbf{r}_{i j}\right|\right)\left[\hat{\mathbf{S}}_{i} \cdot \hat{\mathbf{S}}_{j}-3\left(\hat{\mathbf{S}}_{i} \cdot \mathbf{n}_{i j}\right)\left(\hat{\mathbf{S}}_{j} \cdot \mathbf{n}_{i j}\right)\right],
\end{gathered}
$$

where

$$
d(r)=\frac{\mu_{0} g^{2} \mu_{\mathrm{B}}^{2}}{4 \pi \hbar r^{3}}
$$

$\mathbf{r}_{i j}$ is the vector connecting radicals $i$ and $j$ and $\mathbf{n}_{i j}$ is the unit vector parallel to $\mathbf{r}_{i j}$.

Finally, the exchange Hamiltonian used is as follows:

$$
\hat{H}_{\mathrm{ex}}=\sum_{i<j}^{N} J\left(\left|\mathbf{r}_{i j}\right|\right)\left(\frac{1}{2}+2 \hat{\mathbf{S}}_{i} \cdot \hat{\mathbf{S}}_{j}\right),
$$

where $i$ and $j$ label radicals, and $J\left(\left|\mathbf{r}_{i j}\right|\right)$ is the exchange coupling constant between the $i$ th and $j$ th radicals.

For the work presented here, we have assumed that the system is generated in a random encounter, i.e., $\hat{\rho}(0) \propto \hat{1}$. To calculate the recombination yields, we followed a process similar to that laid out in ref. 64 , i.e., by solving the stochastic Liouville equation of the form:

$$
\frac{\mathrm{d} \hat{\rho}(t)}{\mathrm{d} t}=-i[\hat{H}, \hat{\rho}(t)]-[\hat{K}, \hat{\rho}(t)]_{+}-k_{\mathrm{e}} \hat{\rho}(t),
$$

where the spin-selective recombination is treated using the Haberkorn approach. ${ }^{65,66} k_{\mathrm{e}}$ is the escape rate constant, which accounts for the diffusive separation of the $\mathrm{N}$-radical system 
into the bulk, scavenging and decay processes and all nonrecombination processes in an effective manner; $[\cdot, \cdot]_{+}$indicates the anticommutator and $\hat{K}$ is given by

$$
\hat{K}=\sum_{i, j} \frac{k_{\mathrm{P}}\left(\left|\mathbf{r}_{i j}\right|\right)}{2} \hat{P}_{i j}^{(\mathbf{S})},
$$

where $k_{\mathrm{P}}\left(\left|\mathbf{r}_{i j}\right|\right)$ represents the singlet recombination rate constant between radicals $i$ and $j$ (the left depopulation process at the bottom of in Fig. 1B). The projection operator onto the singlet state is:

$$
\hat{P}_{i j}^{(\mathbf{S})}=\frac{1}{4} \hat{1}-\hat{\mathbf{S}}_{i} \cdot \hat{\mathbf{S}}_{j}
$$

In terms of the operator

$$
\hat{A}=\hat{H}-i \hat{K}
$$

the time-dependent density matrix is given by

$$
\hat{\rho}(t)=\exp \left(-k_{\mathrm{e}} t\right) \exp (-i \hat{A} t) \hat{\rho}(0) \exp \left(+i \hat{A}^{\dagger} t\right) .
$$

Eqn (12) can be evaluated by finding the eigenvalues and eigenvectors of $\hat{A}$. Denoting the former by $\lambda$ and the latter by $\mathbf{T}$, the time integral over $\hat{\rho}(t)$ is given by:

$\int_{0}^{\infty} \hat{\rho}_{i j}(t) \mathrm{d} t=\sum_{k, l, m, n} T_{i, k}\left(T^{-1}\right)_{k, l} \hat{\rho}(0)_{l, m}\left(T^{-1}\right)_{k, m}{ }^{*} T_{j, n} * \frac{1}{k_{\mathrm{e}}+i\left(\lambda_{k}-\lambda_{k}\right)^{*}}$.

From this, we can calculate the recombination yield according to:

$$
\phi_{\mathrm{P}}=2 \operatorname{Tr}\left[\int_{0}^{\infty} \hat{\rho}(t) \mathrm{d} t \hat{K}\right] .
$$

In order to quantify the effect the magnetic field has on the recombination yield, we define the $\operatorname{MFE}\left(\chi_{\mathrm{P}}\right)$, which is calculated by:

$$
\chi_{\mathrm{P}}\left(B_{0}\right)=\frac{\phi_{\mathrm{P}}\left(B_{0}\right)-\phi_{\mathrm{P}}(0)}{\phi_{\mathrm{P}}(0)}
$$

where $\phi_{\mathrm{P}}\left(B_{0}\right)$ is the recombination yield at a magnetic field $B_{0}$ and $\phi_{\mathrm{P}}(0)$ is the recombination yield at zero field.

In general, the MFE depends on the relative position of the radicals and the orientation of the magnetic field. This is a consequence of the dependence of the electron-electron dipolar (EED) interaction on $\mathbf{r}_{i, j}$ as given in eqn (5) and the distance dependence of the exchange coupling constants and recombination rate constants. Here, we assume an exponential distance dependence for the latter parameters:

$$
k_{\mathrm{P}}(r)=k_{\mathrm{P}, 0} \exp (-\beta(r-2 R))
$$

and similarly

$$
J(r)=J_{0} \exp (-\beta(r-2 R)),
$$

with $R$ denoting the lipid radius. We use a common decay length $\beta=1.4 \AA^{-1}$ for all exchange and rate terms.

We estimate that the lateral diffusional displacement of lipids is sufficiently small in a biological membrane to neglect it during the short timespan considered here (100 ns) to give rise to the MFEs. We furthermore estimate that the spin-rotational relaxation of the lipids considered as rigid bodies is slower than the characteristic time for the MFEs, as the rotational tumbling motion is sufficiently slow. See the ESI $\dagger$ for an in-depth discussion of these simplifying assumptions.

\section{Results}

\subsection{Hyperfine coupling constants in lipid peroxyl radicals}

In order to assess the possibility of hyperfine-mediated MFEs in the pairwise recombination of peroxyl radicals (reaction (V) in Fig. 1A), we have calculated the hyperfine coupling constants of selected peroxyl radicals using density functional theory. The basis set EPR-III and the exchange-correlation functional B3LYP have been used; details are summarized in the ESI $\dagger$ (Fig. S1). This analysis was undertaken despite discouraging claims that the hyperfine-derived MFEs are out of the question for $\mathrm{RO}_{2}{ }^{\bullet}$, as the spin density was exclusively concentrated on non-magnetic oxygen atoms. ${ }^{57,62}$ Surprisingly, our study of two peroxyl radicals derived from the unsaturated fatty acid linoleic acid suggests that this is not the case. Marked hyperfine interactions of the order of $10 \mathrm{MHz}$ have been found for the vicinal hydrogen atom, i.e., the hydrogen atom at the location of the peroxyl group. Two different locations of the peroxide group were tested: carbon 9 and 13, and the stereochemistry of the adjacent double bonds was chosen as e and $\mathrm{z}$, and $\mathrm{z}$ and e, respectively (see Fig. 2). Herein we refer to these lipids as $9 \mathrm{ez}$ and 13ze. Based on product analyses, these radicals are expected to be central intermediates of the lipid autoxidation of linoleic acid and its derivative lipids. ${ }^{30}$ We have systematically scanned the dihedral angle that the peroxide group makes with the backbone. This analysis suggests that the rotation is significantly hindered, even at $T=310 \mathrm{~K}$. The high energy configurations are 6.8 and $4.8 \mathrm{~kJ} \mathrm{~mol}^{-1}$ above the most stable rotamers of 9ez and 13ze, respectively. The Boltzmann weighted average hyperfine coupling parameters have been evaluated and are summarized in the ESI. $\dagger$ For 9ez, the dominant hyperfine interaction at the vicinal hydrogen is characterised by a nearly axial tensor with an isotropic value of $13.5 \mathrm{MHz}$ and anisotropy $14.8 \mathrm{MHz}$. For 13ze, the isotropic contribution and the anisotropy evaluate to 10.3 $\mathrm{MHz}$ and 14.6 $\mathrm{MHz}$, respectively. In addition to the vicinal hydrogen atoms, a few of the hydrogen atoms in the neighbourhood of the peroxyl group exhibit small hyperfine interactions (see Fig. 2). The isotropic values of these additional interactions are smaller than $1 \mathrm{MHz}$ in absolute value; the largest principal values approach $6 \mathrm{MHz}$ (see Fig. S1, ESI $\dagger$ ). Below, we will consider the effect of the isotropic hyperfine coupling constant of the vicinal proton on the MFEs. We will for now disregard the anisotropic components for two reasons: firstly, the anisotropies are expected to be reduced by the molecular motion. For example, fast rotation of 13ze about the axis of the carbon chain would reduce the anisotropy from $14.6 \mathrm{MHz}$ to $4.4 \mathrm{MHz}$ (see ESI $\dagger$ for details, Fig. S2 and S3). Secondly, considering anisotropic hyperfine interactions would unreasonably complicate the discussion, which here is focused on qualitative description rather than the quantitative reproduction of a 
A

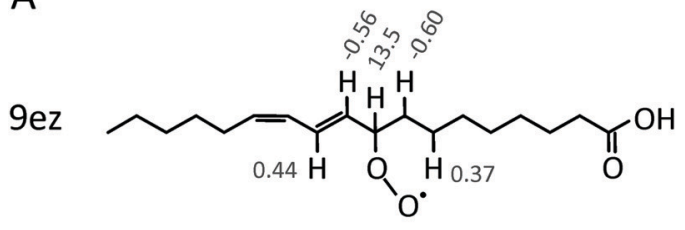

$13 z e$
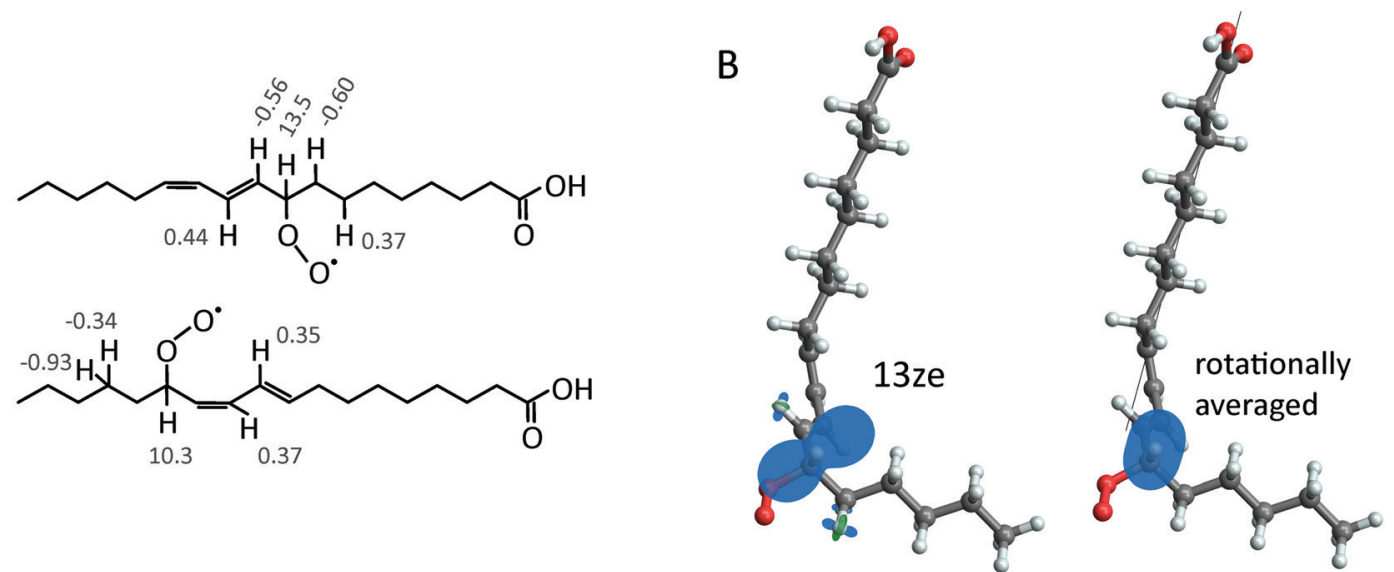

Fig. 2 (A) The structure of the isomers $9 \mathrm{ez}$ (top) and 13ze (bottom) of the linoleic acid peroxyl radical. Labels indicate the Boltzmann-weighted average isotropic hyperfine constant $(T=310 \mathrm{~K}$ ) as the peroxyl group rotates. All coupling constants are shown in $\mathrm{MHz}$. (B) 3D model of the 13ze isomer with graphical representations of the hyperfine interactions. The left structure illustrates the hyperfine coupling constants at $T=310 \mathrm{~K}$; the right structure shows the hyperfine interaction of the vicinal proton under the assumption that fast rotation about the carbon backbone (axis shown as black line) averages the hyperfine interaction (see Fig. S3, ESI $\dagger$ ).

particular MFE. In particular, all calculations would depend on a multitude of parameters describing the relative orientation and their internal and rigid body dynamics. Even if this is desirable for a future study, these parameters cannot currently be described in sufficient detail to warrant this calculation.

\section{2 $\quad$ MFE in radical pairs (RPM)}

In Fig. 3 we summarize the typical field-dependence of the recombination yield for the encounter of two or three $\mathrm{RO}_{2}{ }^{-}$ radicals. Our model calculations followed the general layout as discussed above. In the pair-wise reaction of identical radicals $(N=2)$, MFEs can only ensue from the hyperfine-induced spin evolution. The electron-electron dipole (EED) interaction and the exchange interaction do not mix singlet and triplet states and no magnetosensitivity can ensue from their sole action. However, the large hyperfine interaction from the vicinal proton does provide an unexpected magnetosensitive pathway. This is evident from our model calculations taking into account the isotropic components as derived in the previous section. In fact, if it were the only spin-coupling mechanism (Fig. 3, left, grey lines), large MFEs with equally large low-field effects (LFE) would be expected. The sign of the saturated MFE is negative as expected for an F-pair with dominant recombination efficiency in the singlet configuration, i.e., the magnetosensitive spin dynamics resemble that of the triplet-born radical pair. The LFE results from altered $\mathrm{S}-\mathrm{T}_{0}$-mixing due to altered conservation quantities in the presence and absence of magnetic fields. ${ }^{9,10,67}$ Its large size, as revealed by Fig. 3 (left), is in line with the
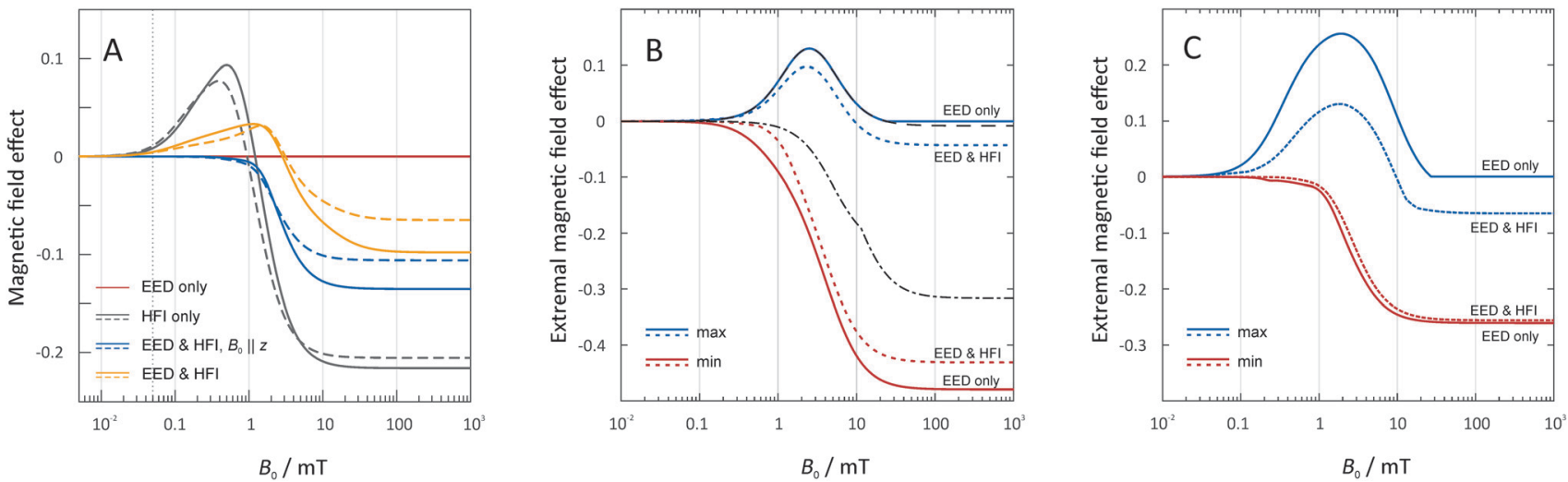

Fig. 3 MFE for two- and three-radical systems. Two radicals are always considered at contact, i.e., at a distance of $9 \AA$, and thus able to recombine. In the three-radical systems (panels B and C), the third radical position is scanned across the membrane plane with excluded volume effects taken into account. For the two-radical system (panel A), dashed lines indicate an isotropic hyperfine constant of $10.3 \mathrm{MHz}$ for the vicinal proton; blue solid lines represent $13.5 \mathrm{MHz}$. Interactions within the Hamiltonian have been included according to the legend. In the three-radical system (panels $B$ and $C$ ), the blue solid lines indicate the maximum MFE with the location of $C$ varied. Similarly, the red solid lines indicate the minimum. Panel $C$ applies to the MFE calculated from the mean recombination yields of systems randomly oriented with respect to the magnetic field. In each case, we considered the exclusion of (solid lines) and inclusion of (dashed lines) the hyperfine coupling with a coupling constant of $10.3 \mathrm{MHz}$ (right). For B, the black dashed and dashes-dotted lines indicate the maximal and minimal (most negative) MFEs resulting from the third spin in contact with the radical pair. $k_{P, 0}=0.2 \mathrm{~ns}^{-1}$ and $k_{\mathrm{e}}=0.01 \mathrm{~ns}^{-1}$. 
simplicity of the spin system as derived above. More complex systems with many coupled nuclear spins typically yield smaller LFEs. ${ }^{68,69}$ For radical pairs in membranes, however, lateral diffusion is slow compared to the spin evolution. Hence the EED is not averaged out by molecular motion and its effect of enlarging the singlet-triplet energy gap has a damping effect on the MFEs. For the magnetic field perpendicular to the membrane plane, the LFE is completely suppressed (Fig. 3, left, blue lines). Conversely, for ensembles of randomly oriented two-radical systems, the LFE is preserved (Fig. 3, left, yellow lines), albeit shifted to larger field intensities. In any case, marked MFEs for $B_{0}<1 \mathrm{mT}$ appear possible. This is particularly true as our assumption of radicals at contact is expected to overestimate the effective electron-electron dipolar coupling. For a magnetic field comparable to the geomagnetic field $(50 \mu \mathrm{T})$, MFEs of $-3 \times$ $10^{-5}$ are predicted by our model (compared to 0.011 without electron-electron dipolar coupling), when using 10.3 $\mathrm{MHz}$ for the hyperfine coupling constant. Effects of the exchange interaction are discussed below.

\subsection{MFE caused or modulated by three-radical interactions}

In three-spin systems, MFEs can ensue even in the absence of hyperfine coupling interactions from the electron-electron dipolar coupling. This effect has been shown to elicit remarkable low-field sensitivity and magnetic field dependencies that can differ from those inflicted by the RPM. ${ }^{29}$ As in the recombination of $\mathrm{RO}_{2}{ }^{\bullet}$ radicals, the dipolar coupling is the dominant interaction, and we expected the D3M to strongly impact upon MFEs in lipid autoxidation. Hence, we have explored this possibility for a range of different scenarios.

In general, we have assumed that two radicals, henceforth referred to as A and B, are at contact and investigated the effect of a third radical, radical $\mathrm{C}$, in relation to its position. Fig. 4 shows the MFE on the recombination yield as a function of the location of $\mathrm{C}$ for the magnetic field oriented perpendicular to the membrane plane. Graphs are displayed for the field intensities $50 \mu \mathrm{T}, 1 \mathrm{mT}$ and $10 \mathrm{mT}$. Additional data for $100 \mathrm{mT}$ are shown in the ESI $\dagger$ (Fig. S4); the MFEs at $100 \mathrm{mT}$ are similar to those at $10 \mathrm{mT}$, however larger. Here, we have placed radical $\mathrm{A}$ at the coordinate $(R, 0)$ and radical $\mathrm{B}$ at $(-R, 0)$ of the membrane plane, with $R=4.5 \AA$ denoting the particle radius. Results are shown for the first quadrant; the MFEs for the other quadrants are related by symmetry. The excluded region surrounding the origin is inaccessible to radical $\mathrm{C}$ (likewise of radius $R$ ) due to the finite size of the lipid radicals.

The left column of Fig. 4 highlights the effect of the dipolar coupling by equating the hyperfine terms to zero. Evidently, large MFE are induced by the mere presence of the third radical. The effects are maximal for radical $\mathrm{C}$ positioned away from contact by approximately $3 \AA$. In particular, isosceles triangular arrangements of the radicals with a triangle height of approximately $12 \AA$ appear to yield large $\left|\chi_{\mathrm{P}}\right|$. For selected positions of radical C, the MFEs are larger than those found for the radical pair model with hyperfine-induced spin mixing. The maximal MFEs amount to $0.063 \%, 9.03 \%$ and $41.9 \%$ in absolute value for the three shown field intensities, $50 \mu \mathrm{T}, 1 \mathrm{mT}$ and $10 \mathrm{mT}$, respectively.

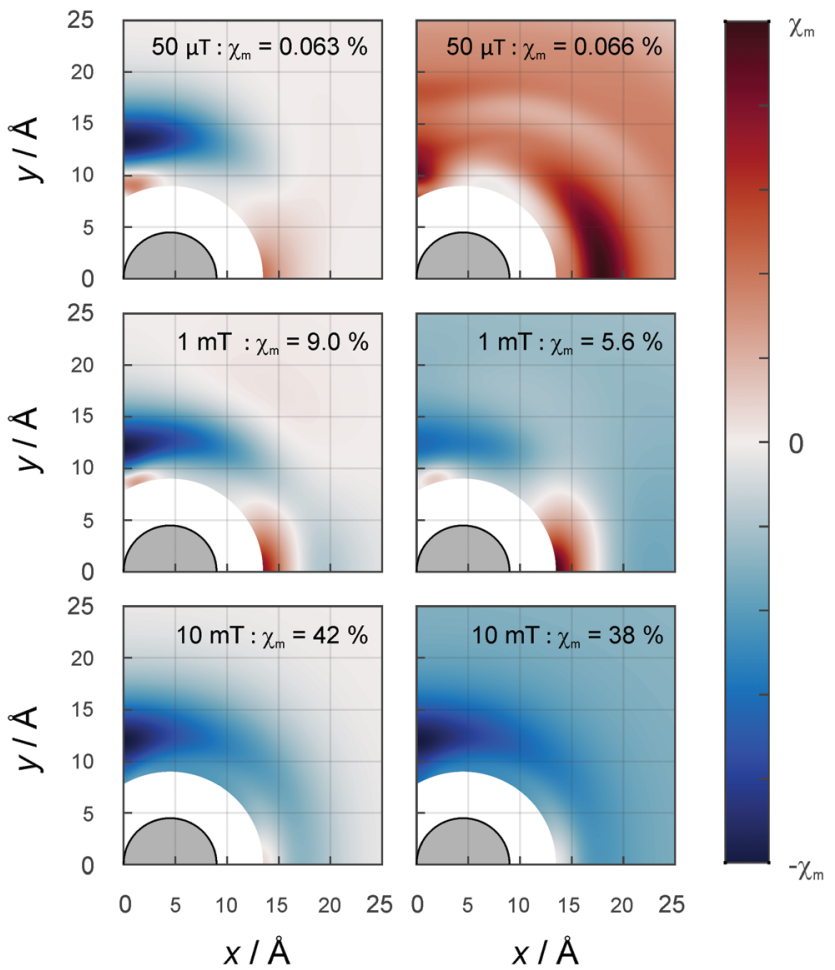

Fig. 4 Dependence of the MFE $\chi_{P}$ in a three-radical system on the position of radical $C$. The vicinal, isotropic hyperfine interaction has been considered (right, $a=10.3 \mathrm{MHz}$ ) or disregarded (left). The magnetic field is perpendicular to the plane of the membrane and the field strengths are (from top to bottom) $50 \mu \mathrm{T}, 1 \mathrm{mT}$ and $10 \mathrm{mT}$. Simulation parameters: $k_{\mathrm{p}, 0}=0.2 \mathrm{~ns}^{-1}$ for all pairs of radicals, $k_{\mathrm{e}}=0.01 \mathrm{~ns}^{-1}$ and $\mathrm{J}=0 . \chi_{\mathrm{m}}$, the maximal absolute value of each MFE, is reported for each sub-panel. The colour bar extends from $-\chi_{m}$ to $\chi_{m}$.

While the figures bring to light the locations of the maximal MFEs, the large effects overwhelm the feature that significant MFEs can also ensue from rather remote $\mathrm{C}$ radicals. For example, for a magnetic field intensity of $10 \mathrm{mT}, \chi_{\mathrm{P}}=-6.5 \%$ results with $\mathrm{C}$ at (20 ̊, $20 \AA$ ), a remarkable $25.3 \AA=5.6 R$ away from the next closest radical. The inherent reactivity of $\mathrm{C}$ is inconsequential everywhere but at the boundary of the excluded volume region. If $\mathrm{C}$ is assumed unreactive, the negative MFEs at this contact region are further enhanced (as is shown in the Fig. S5, ESI $\dagger$ ). A minimal $\chi_{\mathrm{R}}$ of $-63.3 \%$ at $100 \mathrm{mT}$ is then achievable compared to $-47.9 \%$ for reactive $\mathrm{C}$.

If the isotropic hyperfine interactions are re-engaged, the location dependence of the MFE reflects the simultaneous effects of the RPM and the D3M. With the noteworthy exception of the low-field region, the predominant effect of the RPM is the introduce a non-zero MFE when the radical $\mathrm{C}$ is moved great distances away from the radical pair (see Fig. 4, right column for $B_{0} \geq 1 \mathrm{mT}$ ). Other than that, the features of the D3M are still widely present for small $\mathrm{C}$ separations. While the MFEs here again exceed those predicted for the RPM alone, the presence of the hyperfine interactions slightly attenuates the MFEs compared to the D3M-only scenario. In particular, for $B_{0}=100 \mathrm{mT}$ we find a MFE of maximal absolute value of $-43.0 \%$ instead of $-47.9 \%$ from above. This discussion applies 
to moderate to large field intensities $\left(B_{0} \geq 1 \mathrm{mT}\right)$. At $50 \mu \mathrm{T}$ the situation is more interesting: as is evident from Fig. 4 (top right panel), the dipolar coupling with radical $\mathrm{C}$ facilitates comparably large and positive MFEs even for large distances (maximal $\left|\chi_{\mathrm{R}}\right|=0.066 \%$ ); this entails a sign inversion and more than 20 -fold increase in magnitude with respect to the RPM-only MFE $(-0.003 \%)$. The large spatial extent of the dipolar coupling effect is revealed here in the sign change occurring at approximately $44 \AA$, above which the RPM-induced effect becomes dominant. Here, we have assumed $a_{i}=10.3 \mathrm{MHz}$ as suggested for 13ze by our DFT calculations. Qualitatively agreeing conclusions can be drawn for $a_{\text {iso }}=13.5 \mathrm{MHz}$, i.e., 9ez (see Fig. S4, ESI $\dagger$ ).

In order to concisely characterize the field dependence of $\chi_{\mathrm{P}}$ whilst withholding the complexity resulting from the position dependence of the D3M, we shall focus on the extremal (i.e., the largest positive and smallest negative) MFEs. Fig. 3 (middle and right) gives a representation of the supremal and infimal MFEs over all $\mathrm{C}$ locations as a function of the magnetic field intensity. We here focus on two pertinent scenarios: D3M-only $(a=0)$ and the mixed scenario with both the hyperfine interaction (HFI) and EED included. The HFI-only situation is shown in the ESI $\dagger$ (Fig. S6). It merely serves to elaborate on the effect of the hyperfine interaction, but cannot be physically realized as the electronelectron dipolar coupling does not average out by molecular motion in membranes, at least not on the relevant time-scale. Comparing the D3M-only and the mixed-mechanism calculations, it is obvious that in the three-radical systems the electron-electron dipolar coupling is the dominant interaction. While the hyperfine interaction has a small damping effect on the D3M, it also moves the positive MFE-peak to a smaller magnetic field intensity of $1.87 \mathrm{mT}$. Surprisingly, the MFEs generated by the D3M (in the absence or presence of additional hyperfine interactions) can exceed those of the hypothetical HFI-only scenario (Fig. S6, ESI $\dagger$ ), or the effects predicted for isolated radical pairs (Fig. 3, left). In Fig. 3, we have also indicated the minimal and maximal MFE that results from the three radicals in (pairwise) contact (dashed black lines). This analysis suggests that the extremal negative MFEs are generated by the D3M from configurations with radical C offset from the AB-radical pair. On the contrary, the maximal positive MFEs are typically generated with $\mathrm{C}$ in contact with one or both of the radicals. These findings are in line with the exemplary position dependencies shown in Fig. 4.

As the orientational average of the electron-electron dipolar interaction is zero, the question arises to what extent the D3M can elicit MFEs for ensembles of randomly oriented spin systems. The answer to this question is provided by Fig. 5, which shows the dependence of the orientationally averaged MFE on the position of radical C. The data clearly show that substantial MFEs arise from the D3M even for randomly oriented spin systems. For certain relative positions of the radicals, MFEs of the order of $-25 \%$ at $10 \mathrm{mT}$ are possible, both in the absence and in the presence of isotropic hyperfine interactions of $a=10.3 \mathrm{MHz}$. Again, the additional hyperfine interactions have a small damping effect on the MFEs for moderate field intensities. For the low field intensities, "large" effects ( $\approx 4 \%$ at $1 \mathrm{mT}$ ) appear to result from the equilateral triangular geometry. This is especially prominent

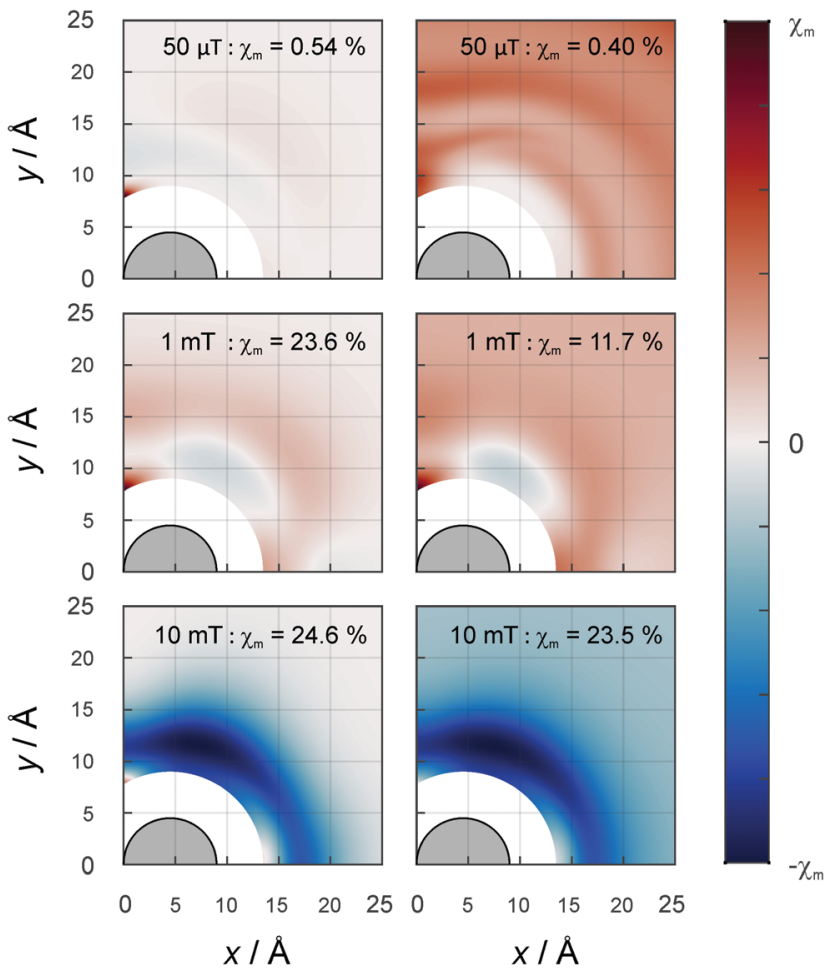

Fig. 5 MFE of the average of the recombination yield when the magnetic field is oriented randomly with respect to the plane of the radicals. Details as for Fig. 4 apply. Without hyperfine (left) and with hyperfine (right, $a=10.3 \mathrm{MHz}$ ), field intensities from top to bottom: $50 \mu \mathrm{T}, 1 \mathrm{mT}$ and $10 \mathrm{mT}$.

for the $50 \mu \mathrm{T}$ magnetic field, but also present at $1 \mathrm{mT}$, in particular for the D3M-only systems. For $50 \mu \mathrm{T}$, in D3M-systems, the MFEs are tiny for all but this particular configuration. Contrarily, if the hyperfine interaction is present as well, MFEs are observed for a wide range of distances. While in this particular case the MFE is predicted to be slightly larger in the isolated radical pair, in general the D3M-induced MFEs exceed those of the RPM in pairs of radicals. For example, for $10 \mathrm{mT}$, three-radical systems can elicit MFEs of $-24.5 \%$ (D3M-only) or $-23.5 \%$ (D3M and HFCs), while $-4.5 \%$ resulted from the isolated, randomly oriented radical pair. In the ESI, $\dagger$ we provide more details on the orientational dependence of the MFE. Here, it suffices to note that the anisotropy of the MFE, i.e., the maximal orientational change relative to the mean MFE, can be substantial in the three-radical systems (see Fig. S7, ESI $\dagger$ ). For the D3M-only systems, anisotropies of $21.5 \%$ and $96.4 \%$ for magnetic fields of $1 \mathrm{mT}$ and $100 \mathrm{mT}$, respectively are found. Fig. 3 (right) illustrates the field dependence of the minimal and maximal MFEs of randomly oriented three-spin systems as a function of the magnetic field. We see that the orientational average favours MFEs at low magnetic fields and the positive MFEs over those found for the $B_{0}$-field perpendicular to the membrane plane. In particular, in the rotationally averaged samples, the MFEs already arise at markedly smaller field intensities than for the perpendicular field direction, $0.03 \mathrm{vs}$. $0.3 \mathrm{mT}$ (compare Fig. 3B and C). Fig. S8 in the ESI $\dagger$ shows the orientation dependence of the field-dependence of the MFE. The onset behaviour is not dissimilar to that observed for 
radical pairs (in the presence of the EED). However, the MFEs can be larger for the D3M by a factor of more than 3 .

\subsection{Effects of the exchange interaction}

The exchange coupling is expected to impact upon the spin dynamics of adjacent radicals. In diradicals in solution, i.e., for averaged electron-electron dipolar interactions but relatively constant exchange interaction, characteristic MFEs can result from the $\mathrm{S} / \mathrm{T}_{ \pm}$-crossing. ${ }^{70,71}$ For immobilized radical pairs, similar degeneracies of the electronic terms can ensue as a function of the dipolar coupling and the external magnetic field. ${ }^{72}$ Here, the exchange coupling could compensate for the non-zero electronelectron dipolar interaction, thereby boosting MFEs and their anisotropies at low magnetic fields. Motivated by these findings, we have analysed the dependence of our model of peroxyl radical recombination in membranes on the exchange coupling interaction. Fig. 6(A) shows the dependency of the MFE of the radical recombination yield for the RPM as a function of the magnetic field and the exchange interaction. The magnetic field was oriented perpendicular to the membrane, i.e., perpendicular to the RP axis. For large $|J|$, the MFE is characterized by the crossing of the $S$ state and a superposition state of $\mathrm{T}_{+}$and $\mathrm{T}_{-}$. Disregarding the hyperfine interaction, the crossing field, $B_{\mathrm{c}}$, can be calculated analytically. We find:

$$
\frac{g \mu_{\mathrm{B}} B_{\mathrm{c}}}{\hbar}=\sqrt{4 J^{2}-\mathrm{d} J-\frac{1}{2} d^{2}}
$$
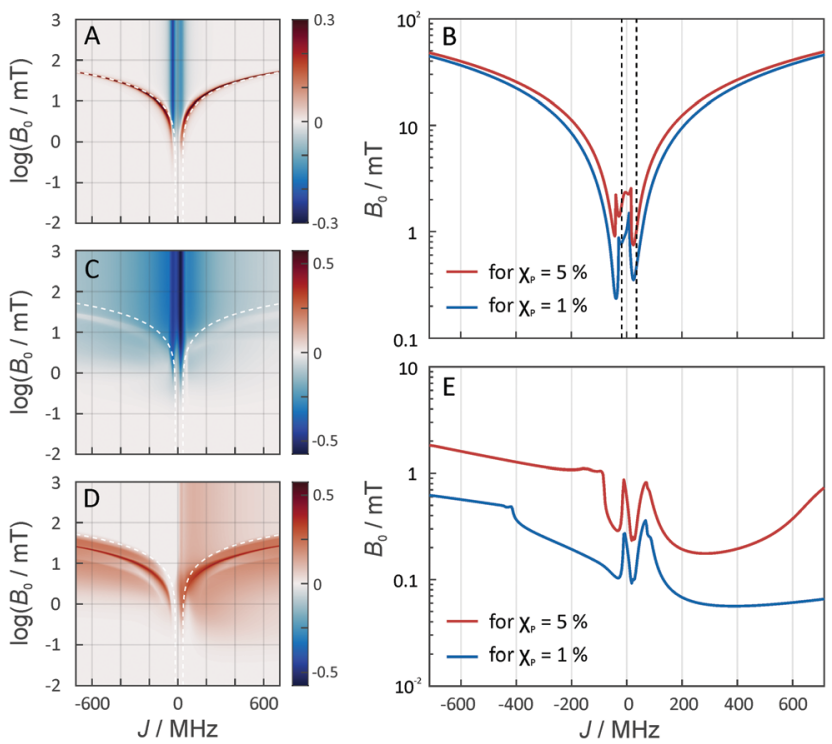

Fig. 6 (A) The MFE for the two-radical radical system when exchange is included. An isotropic hyperfine constant of $10.3 \mathrm{MHz}$ was used and the dipolar interactions were included. $B_{0}$ is perpendicular to the radical axis. (B) The magnetic field required for a MFE of $1 \%$ (red) and $5 \%$ (blue) for the same system as in panel $A$ as a function of the exchange interaction. ( $C$ and D) The minimal and maximal MFEs for the three-radical system when exchange is included. The hyperfine interaction is not included, but the dipolar interactions were taken into account. $\mathbf{B}_{0}$ is perpendicular to the plane made by the three-radicals. (E) The field intensity for a MFE of $1 \%$ (red) and $5 \%$ (blue) for the three-radical system used in (C and D). with $J=J_{\mathrm{A}, \mathrm{B}}$ and $d=d_{\mathrm{A}, \mathrm{B}}>0$ denoting the largest eigenvalue of the electron-electron dipolar coupling tensor. For $B_{\mathrm{c}}=0$, the crossings occur for $J=-\frac{1}{4} d$ and $J=\frac{1}{2} d$, in agreement with, ${ }^{72}$ but using a different convention for the dipolar coupling parameter.

In Fig. $6 \mathrm{~A}, B_{\mathrm{c}}$, as given by eqn (18), has been overlaid on the MFEs (dashed lines). It is apparent that the expression predicts the location of the positive MFEs for larger, in absolute value, exchange coupling constants. For these regions, the involved superposition state of $\mathrm{T}_{+}$and $\mathrm{T}_{-}$, approaches the pure states $\mathrm{T}_{-}$and $\mathrm{T}_{+}$, for positive and negative $J$, respectively, suggesting that the scenario is similar to that for $S / T_{ \pm}$-crossing in the absence of electron-electron dipolar coupling. For smaller $J$, the hyperfine and the exchange coupling have to be treated on an equal footing and complex MFEs ensure. Fig. 6B gives the magnetic field intensities that are necessary to elicit MFEs of $1 \%$ and $5 \%$, regardless of their sign. Two regions of high lowfield sensitivity are evident around $J=-0.53 d$ and $J=+0.35 d$, for which a $1 \%$ MFE can be induced by magnetic fields as small as $0.24 \mathrm{mT}$ and $0.35 \mathrm{mT}$, respectively. For $J=0$, a markedly higher field of $1 \mathrm{mT}$ is necessary.

Fig. 6C-E addresses the $J$-dependence in the three-spin scenario. In order to highlight the effect due to the D3M mechanism, no hyperfine interactions have been considered here. The density plots in Fig. 6C and D give the maximal and minimal MFE realizable through the presence of the third radical. The plots show a positive peak of the MFE for large $|J|$, which resembles that found above for the radical pair scenario and is attributed to a level crossing of $\mathrm{S}$ and $\mathrm{T}_{+} / \mathrm{T}_{-}$-states. However, here, it is shifted to lower field intensities compared to $B_{\mathrm{c}}$, eqn (18), for the radical pair at contact. Furthermore, the MFEs could be larger for the D3M-coupled three-radical system than for the radical pair. What is, however, even more remarkable is the fact that the MFEs in the low-field region do not vanish for large $|J|$. In particular, Fig. 6C and D shows marked MFEs in the $B_{0}<1 \mathrm{mT}$ region regardless of $J$, which are absent from the RPM model, Fig. 6B, except for the two peculiar $J$ s identified above (i.e. $-0.53 d$, $+0.35 d$ ). This is even more prominent in Fig. $6 \mathrm{E}$, which plots the field intensities that are necessary to elicit MFEs of $1 \%$ and $5 \%$ as a function of $J$. This plot reveals the following: firstly, in general, lower magnetic field intensities are necessary to generate a $1 \%$ MFE for the D3M than for the RPM acting on radical pairs. Secondly, large $|J|$ values do not necessarily have a detrimental effect on the low-field sensitivity. E.g., a 1\% effect results from Earth-like magnetic field intensities of $56 \mu \mathrm{T}$ in the presence of a $385 \mathrm{MHz}$ exchange coupling. Any exchange coupling in the range of $200-600 \mathrm{MHz}$ gave rise to similar effects. For negative $J$, slightly larger field intensities are required, which, however, are still smaller than for the RPM. E.g., for $-32 \mathrm{MHz}$, a field intensity of $0.1 \mathrm{mT}$ was required.

\section{Discussion}

D3M offers a pathway to unorthodox MFEs. For systems with non-zero hyperfine interactions, D3M can both boost and 
suppress MFEs relative to those expected from the classical RPM depending on the position of the third radical. For low fields, it can give rise to MFEs of altered sign (such as those shown in Fig. 4 for $B_{0}=50 \mu \mathrm{T}$ ). Unlike the RPM in radical pairs, $\mathrm{D} 3 \mathrm{M}$ is applicable to radicals without hyperfine interactions and for $\Delta g=0$. This is an important realisation, as it suggests MFEs for a large class of radical reactions that have not been assumed magnetosensitive. One system of this kind where this could be important is the recombination of two $\mathrm{RO}_{2}{ }^{\bullet}$ radicals. Here, magnetosensitivity was previously precluded, because of a presumed lack of hyperfine interactions that resulted from the electron density being concentrated on the oxygen atom. ${ }^{57,62}$ This assumption is contrary to our investigation as summarized above, which does suggest significant non-zero hyperfine interactions of the vicinal protons. While this finding does suggest MFEs due to the pairwise RPM, the D3M mechanism might nonetheless be relevant as it can produce stronger MFEs and explain a remarkable low-field sensitivity, which is robust in the presence of large exchange couplings.

As a consequence of the D3M, characteristics of the field dependence of the recombination yield (size of MFEs, width, LFE) will depend on radical concentration. We can estimate the required concentrations for the model of a circular "microreactor" containing a radical pair and an additional radical. We calculate the expectation value of the MFE if the radical and the radical pair are independently distributed over this microreactor while conforming to the excluded volume requirements resulting from the finite size of the radicals:

$$
\begin{aligned}
\bar{\chi}_{\mathrm{r}}= & \frac{1}{V} \int_{0}^{\Omega-R} d_{c} \mathrm{~d} d_{c} \int_{0}^{2 \pi} \mathrm{d} \varphi_{c} \int_{0}^{\pi / 2} \mathrm{~d} \vartheta_{a b} \int_{0}^{\Omega-R} d_{a b} \mathrm{~d} d_{a b} \\
& \times I\left(d_{c}, \varphi_{c}, d_{a b}, \vartheta_{a b}\right) \chi_{\mathrm{r}}\left(\mathbf{r}_{a}\left(d_{a b}, \vartheta_{a b}\right), \mathbf{r}_{b}\left(d_{a b}, \vartheta_{a b}\right), \mathbf{r}_{c}\left(d_{c}, \varphi_{c}\right)\right)
\end{aligned}
$$

where the indicator function $I$ is given by:

$I\left(d_{c}, \varphi_{c}, d_{a b}, \vartheta_{a b}\right)=\Theta\left(2 R-r_{a c}\right) \Theta\left(2 R-r_{b c}\right) \Theta\left(\Omega-R-r_{a}\right) \Theta\left(\Omega-R-r_{b}\right)$,

with $\Theta$ denoting the Heaviside theta function and the location of radical $\mathrm{A}$ and $\mathrm{B}$ in the membrane plane, i.e., $x y$-plane, given by

$$
\mathbf{r}_{a, b}\left(d_{a b}, \vartheta_{a b}\right)=\hat{x}\left(d_{a b} \pm R \cos \vartheta_{a b}\right) \pm \hat{y} R \sin \vartheta_{a b} .
$$

Here, the upper sign refers to radical A and the lower to B. The Cartesian unit vectors have been represented by hatted quantities. The location of radical $\mathrm{C}$ is

$$
\mathbf{r}_{c}\left(d_{c}, \varphi_{c}\right)=\hat{x} d_{c} \cos \varphi_{c}+\hat{y} d_{c} \sin \varphi_{c} .
$$

The indicator function ensures that the radicals are always separated by at least $2 R$ and that the radicals are confined within the microreactor of radius $\Omega . V$ is the phase space volume, i.e., the value of the integral over the indicator function.

Fig. 7 summarizes the dependence of the MFE on the size of the microreactor for $\Omega$ up to $10 R$. For the scenario that the external magnetic field is perpendicular to the membrane plane and that the EED coupling is the only relevant interaction
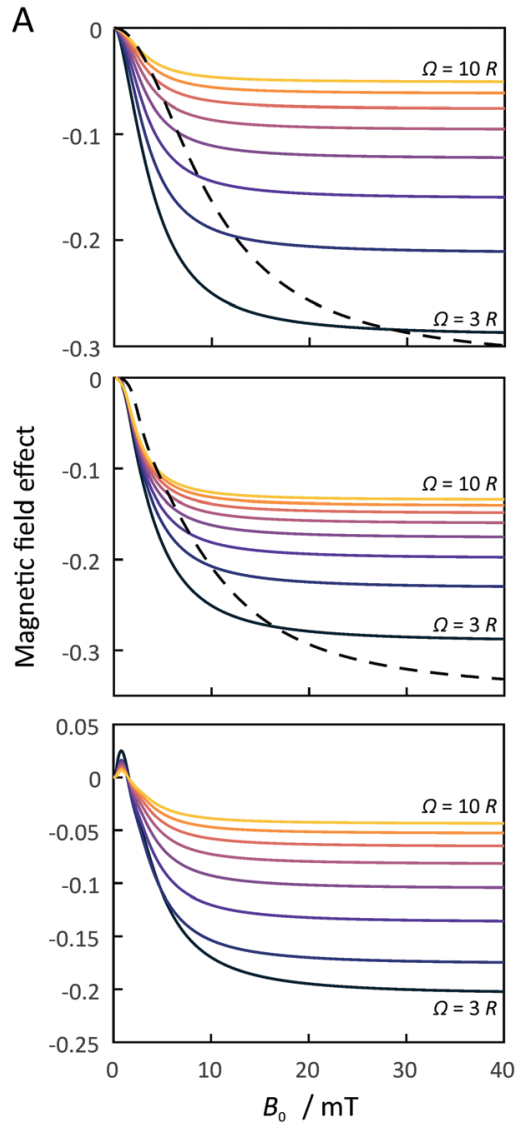

B

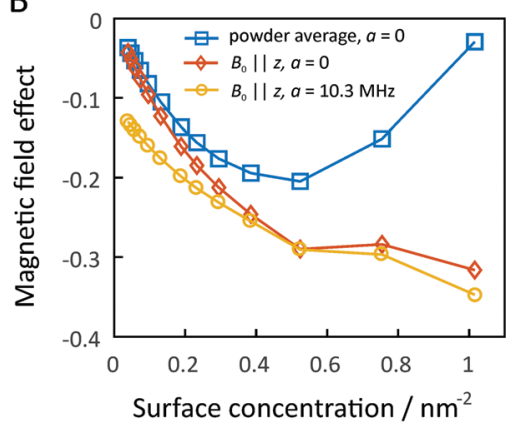

Fig. 7 MFEs resulting from the D3M for circular microreactors. For panel A, the magnetic field of the top and middle rows are perpendicular to the membrane plane. The top row indicates a system without hyperfine interactions, the middle indicates the case when the hyperfine coupling constant in all 3 radicals is $10.3 \mathrm{MHz}$ and the bottom row indicates the orientational average of the top row (dashed lines represent the unique case of the radicals in an equilateral triangle geometry). Panel A scans the magnetic field, and panel B shows the MFEs as a function of the surface concentration. All the other parameters are the same as for Fig. 3.

between the radicals (D3M-only), we find that the MFE decreases to approximately $-30 \%$ as the surface concentration increases to approximately $0.5 \mathrm{~nm}^{-2}$. For the highest possible concentration, which corresponds to an equilateral arrangement of the three radicals in mutual contact within a microreactor of radius $\Omega=\left(\frac{2}{\sqrt{3}+1}\right) R$, the MFE amounts to $-32 \%$. Note, however, that in many respects the MFEs associated with 
the equilateral triangular configuration do not appear as a smooth progression of the MFE at lower surface concentration. It is furthermore noteworthy that the MFE increases faster at low concentrations, i.e., for surface concentrations below $0.15 \mathrm{~nm}^{-2}$, than for the higher concentrations. While large effects are obviously favoured by high concentrations, for $\Omega=10 R$, which corresponds to a surface concentration of merely $0.047 \mathrm{~nm}^{-2}$, a respectable MFE of $-5 \%$ ensues nonetheless. If both EED interactions and HFIs are considered, the MFEs at high surface concentrations resemble those for the D3M-only scenario. At low concentrations, on the other hand, the presence/relevance of the RPM is obvious, which leads to a levelling off of the MFE at $-13 \%$ for small concentrations. The trend of the MFE vs. the microreactor size follows that of the case for the EED system, in that the MFE increases in absolute value as the surface concentration increases. A surface concentration of approximately $0.2 \mathrm{~nm}^{-1}$ leads to the doubling of the MFE over that characteristic for isolated radical pairs and the RPM. For both discussed scenarios the half-saturation widths, i.e., the magnetic field for which half the saturating MFE is achieved, increases with increasing surface concentration (see Fig. S9, ESI $\dagger$ ), but it does so in a different way depending on whether hyperfine interactions are present or not. Furthermore, for both scenarios, no marked low-field feature is obvious. In the ESI, $\dagger$ we also present data for confined three-radical systems subject to the HFI only. For this purely hypothetical scenario, a large low-field effect peaks at $B_{0} \approx 0.36 \mathrm{mT}$ ensues. Its size as well as that of the saturated MFE decreases with increasing surface concentration (see Fig. S11, ESI $\dagger$ ).

We have also extended this analysis to spin systems that are randomly oriented with respect to the external magnetic field (powder average). Remarkably, in this scenario, a feature resembling the LFE of the RPM is evident from the magnetic field dependence even in the absence of HFIs, as is shown in Fig. 7. While this peak of positive MFE resembles that of the hyperfine interaction-derived low-field effect, it here is the result of the EEDmediated process, which gives rise to spiky features at low fields, as anticipated from ref. 29. The low-field peak is located at approximately $0.8 \mathrm{mT}$ and its amplitude increases with increasing surface concentration up to $0.2 \mathrm{~nm}^{-1}$, beyond which it is approximately constant at $2 \%$. At higher fields, the usual response and trends as described above apply. As the saturated MFE grows, in absolute value, faster than the low-field feature, its relative amplitude decreases as the microreactor radius is reduced. Fig. S10 in the ESI $\dagger$ summarizes the characteristics observed for this new kind of LFE.

It is generally remarkable that the third radical can induce effects from a remote distance. The nature of this effect is position- and orientation-dependent, as shown in Fig. 4 and in the ESI $\dagger$ (Fig. S4). Yet, the net effect does not disappear when the powder average of the system is taken; $c f$. Fig. 5. Although assumed in our calculations above, the third radical does not necessarily have to be of the same kind as those forming the reactive radical pair. In particular, more stable radicals such as those derived from radical scavengers could act as the third, i.e., "bystander", radical. Ultimately, this could mean that radical scavengers, while acting to reduce the effect of dangerous free radicals, could promote MFEs via the D3M as they give rise to long-lived radical species upon scavenging ROS, etc. ${ }^{73}$ These MFEs could enhance or counteract the accumulation of free radical damage depending on the relative position, the properties of the individual radicals and the magnetic field intensity, as outlined above.

An important property of the $\mathrm{D} 3 \mathrm{M}$ is the immunisation to large exchange coupling, which is evident in Fig. 6(C-E). This property is expected to be particularly relevant to radical reactions that involve bond formation (and thus positive $J_{\mathrm{ex}}$ ), in systems of reduced mobility, for which no efficient pathways for radical separation and re-encounters are available. This evidently applies to many radical reactions in membranes or other biological environments. For these systems, the average exchange interaction of the reactive radical pair is expected to dominate over magnetic interactions. However, if a third radical were present, comparably large MFEs could be facilitated even for low fields as shown in Fig. 6. This might be one of the reasons why MFEs in biological systems are often found to be associated with conditions of oxidative stress. Such effects are, for example, detailed in the review by Valko et al. ${ }^{74}$ The magnetosensitivity of such reactions could in principle have a significant impact on the long-term risk assessment of magnetic field exposure, as oxidative stress has been linked to the pathogenesis of several human diseases including, notably, cancer. ${ }^{75}$ Clearly, many more experimental and theoretical studies will be necessary to substantiate or refute this possible link. Here, we only provide one more puzzle stone to our understanding of which processes are to be considered in the first place.

As a consequence of the possible dependence of MFEs on concentration, as we propose here, we suggest that study protocols, in particular in the biological context, ought to not only control the exposure conditions, but also regulate and document absolute radical concentrations. Only in this way can the reproducibility of the results be ensured, the underlying MFE be fully characterized and its mechanism unequivocally established. Conversely, it is not inconceivable that the irreproducibility haunting many exposure studies of biological systems resulted from concentrationdependent shifts in the MFE-generating mechanisms.

In principle, interesting MFEs could also result from the dipolar interaction between more than three radicals. While the mechanism only requires passing encounters of radicals, these encounters are obviously progressively less likely as the number of radicals increases. The exceptions to this are reactions in which radicals are generated with high local concentrations, e.g., such as in the context of ionising radiation interacting with biological tissue. In ion-beam therapy, huge local concentrations of hydroxyl radicals ensue in the surroundings of the so-called Bragg peak. ${ }^{76,77}$ While their short lifetime surely required high magnetic fields to induce MFEs, these radical clusters could be subject to MFEs due to a D $n \mathrm{M}$ with $n>3$.

On the whole, our results show that, in theory, unusual MFEs are possible in the oxidative degradation of phospholipids. This could provide an explanation for the unorthodox MFEs observed in ref. 51. Yet, we refrain at this stage from a direct comparison, as high-quality data of the field-dependence of the 
MFE are currently lacking, both, for complex in vivo systems and in vitro models. However, our calculations do reproduce the conclusion that the rate of lipid peroxidation of 1,2-dioleoylphosphatidylcholine liposomes increases even with small fields. ${ }^{33}$

\section{Conclusions}

We have presented model calculations that demonstrate that the recombination of lipid peroxyl radicals is expected to be magnetosensitive. We showed that this magnetosensitivity is underpinned by the classical hyperfine mechanism, i.e., the RPM, as acting in radical encounter pairs, and, if the local radical concentration is sufficient, the dipolarly mediated spin dynamics in groups of three radicals (D3M). Our suggestion of an RPM-based MFE of the symmetric recombination reaction of peroxyl radicals is in deviance with previous suggestions, which opposed this possibility with reference to the supposedly strong localisation of the spin density on the oxygen in the peroxyl groups, which precluded significant hyperfine interactions. Here, we have employed DFT calculations to show that this picture is ill-conceived, significant HFIs are observed for the vicinal proton and, to a lesser extent, a few other protons, and that RPM-derived MFEs are possible. These RPM-MFEs are, however, strongly impacted by the electron-electron dipolar coupling and the exchange interaction in the radical pair at contact, which suppresses marked effects to weak magnetic fields, i.e., the well known low-field effect.

We show that the D3M model, previously introduced in ref. 29, is relevant to the considered scenario of peroxyl radical recombination if the local surface concentration is of the order of $0.1 \mathrm{~nm}^{-1}$. This alternative mechanism has interesting properties: the third radical can induce effects even if it acts only as a remote "bystander", and is not involved in the actual recombination process by means other than its long-range electron-electron dipolar coupling interaction. A large variety of MFEs can result from this interaction, as shown above. The D3M could be particularly relevant for the low-field response of the recombination process. This is the consequence of two surprising effects. Firstly, in the presence of the third radical, the D3M-MFEs are to some degree immunized to the effect of strong exchange interactions, which are expected to reign in the recombination processes under bond-formation. Second, a low-field effect-like feature emerges from the D3M for randomly oriented samples. While this was anticipated based on our previous study of idealized three-radical systems, ${ }^{29}$ it is remarkable that it generalizes to the more realistic conditions (e.g., explicit consideration of recombination) used here and emerges despite the short lifetime of the three-radical system. This result demonstrates that, besides the RPM, the D3M is a second mechanism for a dedicated low-field response of radical reactions. An important conclusion of our study is that MFEs in lipid autoxidation, as well as more generally, could potentially depend on the absolute radical concentrations as a consequence of its impact on the probability of three-radical encounters. This stipulates that studies of the effect of magnetic fields on biological systems should also report radical concentrations and procedures to ensure a constant radical background and antioxidant levels. Any parameter that impacts radical accumulation, e.g., light exposure, ought to be strictly controlled. In this context it is interesting to note that biological MFEs, i.e., MFEs on the level of cells or entire organisms, are often implicated with oxidative stress and/or radical promoting pre-exposures. Here we have established the plausibility of arguments of this form, and it is our hope that further experimental studies along these lines will be conducted, moving us ever closer to a comprehensive understanding of the mysteries of MFEs in biology.

\section{Conflicts of interest}

There are no conflicts to declare.

\section{Acknowledgements}

We thank EPSRC (grant no. EP/R021058/1) for financial support. This project made use of time on ARCHER granted via the UK High-End Computing Consortium for Biomolecular Simulation, HECBioSim (www.hecbiosim.ac.uk), supported by EPSRC (grant no. EP/L000253/1). We are thankful to NVIDIA for supporting this research through their GPU Grant Program. The datasets generated during the current study are available from the corresponding author on reasonable request.

\section{References}

1 U. E. Steiner and T. Ulrich, Magnetic field effects in chemical kinetics and related phenomena, Chem. Rev., 1989, 89(1), 51-147.

2 H. Hayashi, Introduction to Dynamic Spin Chemistry, World Scientific, 2004.

3 K. Maeda, K. Henbest, F. Cintolesi, I. Kuprov, C. Rodgers, P. A. Liddell, D. Gust, C. Timmel and P. J. Hore, Chemical compass model of avian magnetoreception, Nature, 2008, 453, 387-390.

4 P. J. Hore and H. Mouritsen, The radical-pair mechanism of magnetoreception, Annu. Rev. Biophys., 2016, 45, 299-344.

5 M. E. Maffei, Magnetic field effects on plant growth, development, and evolution, Front. Recent Dev. Plant Sci., 2014, 5, 445.

6 E. W. Evans, D. R. Kattnig, K. B. Henbest, P. J. Hore, S. R. Mackenzie and C. R. Timmel, Sub-millitesla magnetic field effects on the recombination reaction of flavin and ascorbic acid radicals, J. Chem. Phys., 2016, 145(8), 085101.

7 C. T. Rodgers and P. J. Hore, Chemical magnetoreception in birds: the radical pair mechanism, PNAS, 2009, 106(2), 353-360.

8 U. Steiner, K. M. Salikhov, Yu. N. Molin, R. Z. Sagdeev, A. L. Buchachenko: Spin Polarization and Magnetic Effects in Radical Reactions, vol. 22 aus "Studies in Physical and Theoretical Chemistry", Elsevier Scientific Publ. Comp., Amsterdam, Oxford, New York, Tokyo 1984. 419 Seiten, Preis: DM 225, Ber. Dtsch. Chem. Ges., 1984, 88(11), 1168B-1169B.

9 C. R. Timmel, U. Till, B. Brocklehurst, K. A. Mclauchlan and P. J. Hore, Effects of weak magnetic fields on free radical recombination reactions, Mol. Phys., 1998, 95(1), 71-89. 
10 A. M. Lewis, T. P. Fay, D. E. Manolopoulos, C. Kerpal, S. Richert and C. R. Timmel, On the low magnetic field effect in radical pair reactions, J. Chem. Phys., 2018, 149(3), 034103.

11 B. Brocklehurst, Magnetic fields and radical reactions: recent developments and their role in nature, Chem. Soc. Rev., 2002, 31, 301-311.

12 C. T. Rodgers, Magnetic field effects in chemical systems, Pure Appl. Chem., 2009, 81(1), 19-43.

13 H. M. Hoang, V. Pham, G. Grampp and D. R. Kattnig, Magnetic field-sensitive radial pair dynamics in polymethylene ether-bridged donor-acceptor systems, ACS Omega, 2018, 3, 10296-10305.

14 E. Ciejka, P. Kleniewska, B. Skibska and A. Goraca, Effects of extremely low frequency magnetic field on oxidative balance in brain of rats, J. Physiol. Pharmacol., 2011, 62, 657-661.

15 D. R. Kattnig, E. Evans, V. Dejean, C. Dodson, M. Wallace, S. Mackenzie, C. Timmel and P. J. Hore, Chemical amplification of magnetic field effects relevant to avian magnetoreception, Nat. Chem., 2016, 8, 384-391.

16 H. M. Hoang, T. B. V. Pham, G. Grampp and D. R. Kattnig, Exciplexes versus loose ion pairs: How does the driving force impact the initial product ratio of photoinduced charge separation reactions?, J. Phys. Chem. Lett., 2014, 5(18), 3188-3194.

17 T. Ritz, S. Adem and K. Schulten, A model for photoreceptorbased magnetoreception in birds, Biophys. J., 2000, 78(2), 707-718.

18 D. R. Kattnig, I. A. Solov'yov and P. J. Hore, Electron spin relaxation in cryptochrome-based magnetoreception, Phys. Chem. Chem. Phys., 2016, 18, 12443-12456.

19 E. M. Gauger, E. Rieper, J. J. Morton, S. C. Benjamin and V. Vedral, Sustained quantum coherence and entanglement in the avian compass, Phys. Rev. Lett., 2011, 106(4), 040503.

20 D. Kattnig, J. Sowa, I. A. Solov'yov and P. J. Hore, Electron spin relaxation can enhance the performance of a cryptochromebased magnetic compass sensor, New J. Phys., 2016, 18, 063007.

21 H. G. Hiscock, S. Bourne-Worster, D. Kattnig, C. Steers, Y. Jin, D. Manolopoulos, H. Mouritsen and P. J. Hore, The quantum needle of the avian magnetic compass, Proc. Natl. Acad. Sci. U. S. A., 2016, 113, 201600341.

22 A. Marais, B. Adams, A. K. Ringsmuth, M. Ferretti, J. M. Gruber, R. Hendrikx, M. Schuld, S. L. Smith, I. Sinayskiy, T. P. J. Krüger, F. Petruccione and R. van Grondelle, The future of quantum biology, J. R. Soc., Interface, 2018, 15(148), 20180640.

23 A. L. Buchachenko and V. L. Berdinsky, Electron spin catalysis, Chem. Rev., 2002, 102(3), 603-612.

24 D. R. Kattnig and P. J. Hore, The sensitivity of a radical pair compass magnetoreceptor can be significantly amplified by radical scavengers, Sci. Rep., 2017, 7, 11640.

25 D. R. Kattnig, Radical-pair-based magnetoreception amplified by radical scavenging: Resilience to spin relaxation, J. Phys. Chem. B, 2017, 121(44), 10215-10227.

26 A. S. Letuta and V. L. Berdinskii, Chemical zeno effect - a new mechanism of spin catalysis in radical triads, Dokl. Phys. Chem., 2015, 463(2), 179-181.

27 H. J. Hogben, O. Efimova, N. Wagner-Rundell, C. R. Timmel and P. J. Hore, Possible involvement of superoxide and dioxygen with cryptochrome in avian magnetoreception: origin of Zeeman resonances observed by in vivo epr spectroscopy, Chem. Phys. Lett., 2009, 480(1), 118-122.

28 T. Yu. Karogodina, S. V. Sergeeva, D. V. Stass and U. E. Steiner, Magnetic field effect on recombination of nitric oxide and superoxide anion in high magnetic field, Dokl. Phys. Chem., 2011, 436(1), 5-7.

29 R. H. Keens, S. Bedkihal and D. R. Kattnig, Magnetosensitivity in dipolarly coupled three-spin systems, Phys. Rev. Lett., 2018, 121, 096001.

30 G. Spiteller, Linoleic acid peroxidation - the dominant lipid peroxidation process in low density lipoprotein - and its relationship to chronic diseases, Chem. Phys. Lipids, 1998, 95(2), 105-162.

31 C. Mylonas and D. Kouretas, Lipid peroxidation and tissue damage, In Vivo, 1999, 13(3), 295-309.

32 A. Doktorov, N. N. Lukzen and J. Pedersen, Analysis of lipid peroxidation kinetics. 1. role of recombination of alkyl and peroxyl radicals, J. Phys. Chem. B, 2008, 112, 11854-11861.

33 U. Lalo, Y. Pankratov and O. Mykhaylyk, Steady magnetic-fields effect on lipid-peroxidation kinetics, Redox Rep., 1994, 1, 71-75.

34 E. Ciejka and A. Goraca, Influence of low magnetic field on lipid peroxidation, Pol. Merkuriusz Lek., 2008, 24, 106-108.

35 Y. I. Novitskii, G. V. Novitskaya, Y. A. Serdyukov, T. K. Kocheshkova, D. R. Molokanov and M. V. Dobrovolskii, Influence of weak permanent magnetic field on lipid peroxidation in radish seedlings, Russ. J. Plant Physiol., 2015, 62(3), 375-380.

36 M. Giorgio, M. Trinei, E. Migliaccio and P. G. Pelicci, Hydrogen peroxide: a metabolic by-product or a common mediator of ageing signals?, Nat. Rev. Mol. Cell Biol., 2007, 8, 722A-728A.

37 M. M. Gaschler and B. R. Stockwell, Lipid peroxidation in cell death, Biochem. Biophys. Res. Commun., 2017, 482(2), 419-425.

38 N. Hogg and B. Kalyanaraman, Nitric oxide and lipid peroxidation, Biochim. Biophys. Acta, 1999, 1411, 378-384.

39 I. Tejero, À. González-Lafont, J. M. Lluch and L. A. Eriksson, Theoretical modeling of hydroxyl-radical-induced lipid peroxidation reactions, J. Phys. Chem. B, 2007, 111(20), 5684-5693.

40 J. M. C. Gutteridge, Iron promoters of the Fenton reaction and lipid peroxidation can be released from haemoglobin by peroxides, FEBS Lett., 1986, 201, 291-295.

41 I. F. Benzie, Lipid peroxidation: a review of causes, consequences, measurement and dietary influences, Int. J. Food Sci. Nutr., 1996, 47, 233-261.

42 E. E. Farmer and M. J. Mueller, ROS-mediated lipid peroxidation and res-activated signalling, Annu. Rev. Plant Biol., 2013, 64, 429-450.

43 H. Yin, L. Xu and N. A. Porter, Free radical lipid peroxidation: mechanisms and analysis, Chem. Rev., 2011, 111, 5944-5972.

44 H. Okano, Effects of static magnetic field in biology: role of free radicals, Front. Biosci., 2008, 13, 6106-6125.

45 S. Ghodbane, A. Lahbib, M. Sakly and H. Abdelmelek, Bioeffects of static magnetic fields: oxidative stress, genotoxic effects, and cancer studies, BioMed Res. Int., 2013, 2013, 602987.

46 D. A. Pratt, K. A. Tallman and N. A. Porter, Free radical oxidation of polyunsaturated lipids: new mechanistic 
insights and the development of peroxyl radical clocks, Acc. Chem. Res., 2011, 44(6), 458-467.

47 P. Mamrot, P. Politanski, M. Zmyslony, E. Rajkowska and J. Jajte, The effect of weak $50 \mathrm{hz}$ magnetic field on the number of free oxygen radicals in rat lymphocytes in vitro, Bioelectromagnetics, 2004, 25, 607-612.

48 J. Jajte, J. Grzegorczyk, M. Zmyslony and E. Rajkowska, Effect of $7 \mathrm{mT}$ static magnetic field and iron ions on rat lymphocytes: apoptosis, necrosis and free radical processes, Bioelectrochemistry, 2002, 57(2), 107-111.

49 S. Coskun, B. Balabanl, A. Canseven and N. Seyhan, Effects of continuous and intermittent magnetic fields on oxidative parameters textit in vivo, Neurochem. Res., 2008, 34, 238-243.

50 B. Lee, H. Johng, J. Lim, J. H. Jeong, K. Y. Baik, T. J. Nam, J. H. Lee, J. Kim, U. D. Sohn, G. Yoon, S. Shin and K. Soh, Effects of extremely low frequency magnetic field on the antioxidant defense system in mouse brain: a chemiluminescence study, J. Photochem. Photobiol., B, 2004, 73(1), 43-48.

51 H. Kabuto, I. Yokoi, N. Ogawa, A. Mori and R. P. Liburdy, Effects of magnetic fields on the accumulation of thiobarbituric acid reactive substances induced by iron salt and $\mathrm{H}_{2} \mathrm{O}_{2}$ in mouse brain homogenates or phosphotidylcholine, Pathophysiology, 2001, 7(4), 283-288.

52 R. Hanini, A. Chatti, S. B. Ghorbel and A. Landoulsi, Role of SOD gene in response to static magnetic fields in Pseudomonas aeruginosa, Curr. Microbiol., 2017, 74, 05.

53 G.-R. Ding, T. Nakahara, H. Hirose, S. Koyama, Y. Takashima and J. Miyakoshi, Extremely low frequency magnetic fields and the promotion of $\mathrm{H}_{2} \mathrm{O}_{2}$-induced cell death in HL-60 cells, Int. J. Radiat. Biol., 2004, 80(4), 317-324.

54 M. Inotani, S. Fukuyoshi and T. Kusumi, Autoxidation of linoleic acid in a strong magnetic field (9.4 t), Tetrahedron Lett., 2001, 42, 7451-7452.

55 E. Gizem Kvrak, K. Kübra Yurt, A. A. Kaplan, I. Alkan and G. Altun, Effects of electromagnetic fields exposure on the antioxidant defense system, J. Microsc. Ultrastruct., 2017, 5(4), 167-176.

56 N. M. Emanuel and A. B. Gagarina, Critical phenomena in chain branching reactions with degenerate chain branching, Russ. Chem. Rev., 1966, 35(4), 260.

57 A. Kipriyanov Jr., A. Doktorov and P. Purtov, Magnetic field effects on bistability and bifurcation phenomena in lipid peroxidation, Bioelectromagnetics, 2015, 36(7), 485-493.

58 E. M. Pliss, A. M. Grobov, A. K. Kuzaev and A. L. Buchachenko, Magnetic field effect on the oxidation of hydrocarbons by molecular oxygen, Mendeleev Commun., 2017, 27(3), 246-247.

59 R. Kohen and A. Nyska, Oxidation of biological systems: Oxidative stress phenomena, antioxidants, redox reactions, and method for their quantification, Toxicol. Pathol., 2002, 30, 620-650.

60 G. A. Russell, Deuterium-isotope effects in the autoxidation of aralkyl hydrocarbons. mechanism of the interaction of peroxy radicals, J. Am. Chem. Soc., 1957, 79(14), 3871-3877.

61 F. J. Adrian, Principles of the radical pair mechanism of chemically induced nuclear and electron spin polarization, Res. Chem. Intermed., 1979, 3, 3-43.
62 E. M. Pliss, A. M. Grobov, A. K. Kuzaev and A. L. Buchachenko, Magnetic field effect on the oxidation of hydrocarbons by molecular oxygen, Mendeleev Commun., 2017, 27, 246-247.

63 R. D. Hills Jr. and N. McGlinchey, Model parameters for simulation of physiological lipids, J. Comput. Chem., 2016, 37(12), 1112-1118.

64 D. V. Sosnovsky, G. Jeschke, J. Matysik, H.-M. Vieth and K. L. Ivanov, Level crossing analysis of chemically induced dynamic nuclear polarization: towards a common description of liquid-state and solid-state cases, J. Chem. Phys., 2016, 144(14), 144202.

65 R. Haberkorn, Density matrix description of spin-selective radical pair reactions, Mol. Phys., 1976, 32(5), 1491-1493.

66 T. P. Fay, L. P. Lindoy and D. E. Manolopoulos, Spin-selective electron transfer reactions of radical pairs: beyond the Haberkorn master equation, J. Chem. Phys., 2018, 149(6), 064107.

67 A. M. Lewis, T. P. Fay, D. E. Manolopoulos, C. Kerpal, S. Richert and C. R. Timmel, On the low magnetic field effect in radical pair reactions, J. Chem. Phys., 2018, $149(3), 034103$.

68 E. W. Evans, D. R. Kattnig, K. B. Henbest, P. J. Hore, S. R. Mackenzie and C. R. Timmel, Sub-millitesla magnetic field effects on the recombination reaction of flavin and ascorbic acid radicals, J. Chem. Phys., 2016, 145(8), 085101.

69 C. T. Rodgers, S. A. Norman, K. B. Henbest, C. R. Timmel and P. J. Hore, Determination of radical re-encounter probability distributions from magnetic field effects on reaction yields, J. Am. Chem. Soc., 2007, 129(21), 6746-6755.

70 N. J. Turro and B. Kraeutler, Magnetic field and magnetic isotope effects in organic photochemical reactions. A novel probe of reaction mechanisms and a method for enrichment of magnetic isotopes, Acc. Chem. Res., 1980, 13(10), 369-377.

71 U. E. Steiner, J. Schäfer, N. N. Lukzen and C. Lambert, $\mathrm{J}$-resonance line shape of magnetic field-affected reaction yield spectrum from charge recombination in a linked donoracceptor dyad, J. Phys. Chem. C, 2018, 122(22), 11701-11708.

72 O. Efimova and P. J. Hore, Role of exchange and dipolar interactions in the radical pair model of the avian magnetic compass, Biophys. J., 2008, 94, 1565-1574.

73 V. Lobo, A. Patil, A. Phatak and N. Chandra, Free radicals, antioxidants and functional foods: impact on human health, Pharmacogn. Rev., 2010, 4, 118-126.

74 M. Valko, D. Leibfritz, J. Moncol, M. T. Cronin, M. Mazur and J. Telser, Free radicals and antioxidants in normal physiological functions and human disease, Int. J. Biochem. Cell Biol., 2007, 39(1), 44-84.

75 F. Barnes and B. Greenebaum, Role of radical pairs and feedback in weak radio frequency field effects on biological systems, Environ. Res., 2018, 163, 165-170.

76 O. Desouky and G. Zhou, Biophysical and radiobiological aspects of heavy charged particles, J. Taibah Univ., Sci., 2016, 10(2), 187-194.

77 K. Haume, P. de Vera, A. Verkhovtsev, E. Surdutovich, N. J. Mason and A. V. Solov'yov, Transport of secondary electrons through coatings of ion-irradiated metallic nanoparticles, Eur. Phys. J. D, 2018, 72, 116. 